\title{
El mito americano en la obra de Cesare Pavese y su herencia en la canción de autor italiana de los años setenta
}

\author{
LEONARDO VILEI \\ Universidad Nacional de Educación Distancia \\ lvilei@inwind.it
}

\section{Resumen}

En los años treinta del siglo pasado, una generación entera de escritores e intelectuales italianos encontró la vía para huir de la cerrazón oficial del fascismo a través de la cultura estadounidense. Cesare Pavese estaba entre los principales protagonistas de esta renovación cultural. Gracias a las traducciones al italiano realizadas por los escritores de la Italia fascista de aquellos años, los literatos norteamericanos más importantes: Melville, Faulkner, Dos Passos... fueron conocidos en la península italiana. Fernanda Pivano, amiga personal de Pavese, mantuvo vivo, durante muchos años, el encuentro cultural entre Italia y Estados Unidos, no solo en el ámbito literario, sino también en el musical. Su aportación es evidente en distintos cantautores italianos de los años setenta, como, por ejemplo, en el genovés Fabrizio De André.

Palabras clave: Pavese, literatura americana, fascismo, Pivano, cantautores, De André.

The american myth in the work of Cesare Pavese and his inheritance in the Italian song of the seventies

\begin{abstract}
In the 1930s a generation of Italian writers and intellectuals looked towards the North American culture to escape from the hardship of the Fascist officiality. Cesare Pavese was a main character of this stage, through several translations (Melville, Fawlkner, among others) and essays on American literature. A young Fernanda Pivano, through her friendship with Pavese, continued this work, maintaining alive in Italy the encounter with the American culture, both in literature and in popular music. Her influence can be detected in the music of many songwriter, as Fabrizio De André.
\end{abstract}

Key words Pavese, American literature, Fascism, Pivano, songwriters, De Andrè.

Vilei, Leonardo. 2011. El mito americano en la obra de Cesare Pavese y su herencia en la canción de autor italiana de los años setenta. Cuadernos de Filología Italiana, $\mathrm{n}^{\mathrm{o}}$ extraordinario: págs. 369-375. 
«Tradurre Moby Dick è un mettersi al corrente con i tempi» (Melville 1995: 11). Se abre con esta frase la introducción que Cesare Pavese escribió para la obra maestra de Herman Melville, traducida por él mismo y por primera vez publicada en Italia en 1941, en la cumbre del interés americano demostrado por los más importantes intelectuales y escritores italianos de la época. El interés de Pavese hacia la cultura estadounidense nace muy pronto: desde los años de los estudios universitarios fue, en efecto, apasionado de las películas americanas de aventura, de las que conocía perfectamente directores, actores y actrices ${ }^{1}$. Esta pasión se trasladó en poco tiempo a la literatura, hasta elegir, como objeto de su tesis de licenciatura, por otro lado muy polémica, el análisis de la poesía de Walt Whitman.

En aquellos años, además, empieza a traducir a numerosos escritores americanos -el predilecto desde el principio fue Melville- y en 1931 se imprime en Florencia su primera traducción, Il nostro signor Wrenn de Sinclair Lewis, primer autor estadounidense en haber conseguido el premio Nobel.

La relación con los escritores americanos fue, en Italia en general, a partir de los años treinta del siglo pasado, un hecho cultural y generacional que indudablemente se tiene que relacionar con el Fascismo, como una tentativa de fuga con respecto a una vida cultural escayolada en una oficialidad insufriblemente patriótica:

Eravamo il Paese della risorta romanità dove persino i geometri studiavano il latino [...]. Il sapore di scandalo e di facile eresia che avvolgeva i nuovi libri ed i loro argomenti, il furore di rivolta e di sincerità che anche i più sventati sentivano pulsare in quelle pagine tradotte, riuscirono irrestitibili a un pubblico non ancora del tutto intontito dal conformismo e dall'accademia (Pavese 1962: 174).

En todo caso, la necesitad de huir de la estrechez de la cultura fascista no aclara completamente una experiencia que mucho tuvo que ver con el aprendizaje de algunos entre los más grandes escritores de la época, que tuvieron en común un rasgo que cada generación posee frente a la cultura oficial, es decir, la juventud, tema por otro lado muy importante en toda la obra de Pavese.

En la cita anterior encontramos una palabra, furore, que de inmediato trae a la memoria la obra maestra del otro gran "descubridor" de América, Elio Vittorini ${ }^{2}$. En el célebre incipit de su novela Conversazione in Sicilia - «Io ero, in quell'inverno, in preda agli astratti furori» (Vittorini 1986: 4) - se encuentra la fórmula perfecta que, en la literatura italiana, expresó la condición de un malestar generacional. A partir de este malestar nace el encuentro con los escritores norteamericanos, que

\footnotetext{
1 Sobre el interés cinematográfico de Pavese vid. Lajolo (1972).

2 Vittorini selecciona, comenta y en parte traduce, una serie de cuentos de escritores americanos en una antología titulada Americana, publicada por el editor Valentino Bompiani. Sin embargo, la publicación del libro encuentra muchas dificultades. El régimen fascista impone su censura y solo permite la publicación a pacto de que sean eliminadas todas las notas del autor; además, la introdución de Vittorini hubiera tenido que ser reemplazada por una introducción del escritor Emilio Cecchi, que modificaba drásticamente el sentido de espontaneidad y rebelión que el escritor siciliano vio en mucha literatura norteamericana. Las opiniones de Cecchi serán criticadas por el joven literato antifascista Giame Pintor, según el cual la visión propuesta en la versión modificada por la censura, fue la de una América materialista y sin ideales, en antítesis por lo tanto con el pensamiento de Vittorini y de los demás intelectuales de la nueva generación.
} 
proporciona una posible respuesta cultural antifascista. La profesión de traductor tiene, en este sentido, una gran importancia en la vida de Pavese y de otros escritores de su época, por lo que se refiere a su aprendizaje en ámbito literario, hasta el punto de haber contribuido a abrir un período nuevo en la narrativa italiana.

En los dos artículos que escribe como introducción a las obras de Lewis y Anderson resulta clara la búsqueda de Pavese. En el primero, que lleva el significativo título Senza provinciali una letteratura non ha nerbo (Pavese 1962: 10) cuando Pavese pone de relieve los dos méritos más grandes de Lewis -es decir, el localismo de los personajes y el estilo de la nueva lengua americana, el argot- declara implícitamente el propio programa literario: búsqueda de lo local, inserción de la lengua hablada en la escrita, ruptura con la tradición académica nacional.

En el artículo dedicado a Anderson, Middle West e Piemonte, el escritor, además, propone un paralelismo entre Italia y América, subrayando lo que los italianos, y sobre todo los piamonteses, han intentado en vano hacer. Se trata de alcanzar lo universal a través del descubrimiento y del ahondamiento de los caracteres regionales, algo que los escritores americanos ya lograron en sus novelas. Pavese, por lo tanto, afirma que en las novelas americanas está también indicada la dirección que los literatos italianos deberían seguir:

Non il dialetto crudo ancora troppo locale - come fanno qui da noi gli specialisti dialettali che, anche negli esempi più insigni, conservano sempre qualcosa di un po' gretto - ma una nuova intramatura dell'inglese, tutta fatta di idiotismi americani, di uno stile che non è più il dialetto, ma linguaggio, ripensato, ricreato, poesia. (Pavese 1962: 43).

Las palabras de Pavese no constituyen evidentemente un programa literario válido para todos los protagonistas de este grupo de «americanos»: el carácter generacional, ciertamente importante, no marca el mismo destino para todos. Para cada escritor, la relación con América marca un recorrido literario con resultados distintos, ya visible en las elecciones estilísticas adoptadas en las traducciones.

Se ha hablado a menudo, con intención de acusación, de un Pavese que copia a los norteamericanos -en referencia, en especial modo a Paesi tuoi, su primera obra literaria- o de un Vittorini cargado por el trabajo de traductor e incapaz de deshacerse de las huellas de esta labor. Ciertamente, cada experiencia que contribuye a la reflexión sobre los medios y las posibilidades de la escritura, como es la de la traducción, o el debate cultural de una época, deja una huella sobre el estilo y sobre la formación de los escritores. Sin embargo, es posible identificar las señas de la futura personalidad en las pruebas de aprendizaje, en aquellas traducciones que presentaron problemas de estilo y de lengua y cuyas soluciones formales no fueron las mismas para todos. Especialmente interesante es, por ejemplo, observar cómo Vittorini ${ }^{3}$ y Pavese ${ }^{4}$ tradujeron a Faulkner: el primero casi palabra por palabra, con riguroso respeto de la cadencia, el segundo con un estilo propio ${ }^{5}$.

\footnotetext{
3 Se trata de la novela Luce d'agosto, publicada en 1939 por Mondadori.

4 Se trata de Il borgo, publicada en 1942 por Mondadori.

5 Véase en este sentido el juicio de Adriana Motti, traductora de las más prolíficas en Italia: «A me piaceva più il primo. Pavese era troppo Pavese e Faulkner diventava un po' uno delle Langhe». Cit. en $L$. Sofri, Wittgenstein, en http://www.wittgenstein.it/html/diario000999.html.
} 
La importancia de la personalidad de la traducción es, para Pavese, un factor que hay que tener en cuenta en el momento de reseñar una novela o una colección de poemas. Esta atención hacia la relevancia de la traducción de una obra literaria guarda relación con los años que el escritor dedicó a este trabajo, labor que, sabemos, es muy a menudo escondida o poco valorada por los críticos. Tenemos una muestra de esta actitud de Pavese en I morti di Spoon River, una reseña sobre la traducción que Fernanda Pivano hizo de la obra maestra de Edgar Lee Master. En este artículo el escritor piamontés explica su idea acerca de la aportación del traductor sobre el resultado final:

Ci sarà permesso dire, a questo punto, che invidiamo alla traduttrice lo schietto piacere da lei provato in questa fatica. Di esso testimonia la felicità espressiva, non di rado creatrice, di cui sono pervase non poche di queste pagine. Soltanto chi conosce la sorniona laconicità del testo, tutta intessuta di richiami dialettali, sottintesi di costume, liriche dissonanze e impennamenti, può misurare la resa -l'alchimia- del lavoro compiuto (Pavese 1962: 67).

Esta reseña es doblemente importante, no sólo por lo que acabamos de poner de relieve, sino también porque nos ofrece la prueba de una amistad intelectual muy importante en la cultura italiana. Fernanda Pivano fue traductora, estudiosa y apasionada de la cultura norteamericana y, a lo largo de más de cincuenta años, ha llevado a cabo una labor importantísima de introducción del mundo literario y musical estadounidense en Italia. Cuando ella, joven y entusiasta estudiante universitaria, recoge la herencia de la generación de Pavese, de Vittorini, de Pintor, de alguna manera el mito americano se está extinguiendo, o quizás modificando, en concomitancia con el fin del fascismo. De este declive el mismo Pavese se hace intérprete:

Sono finiti i tempi in cui scoprivamo l'America. [...]. Ora l'America, la grande cultura americana, sono state scoperte e riconosciute, e si può prevedere che per qualche decennio non ci verrà più da quel popolo nulla di simile ai nomi e alle rivelazioni che entusiasmarono la nostra giovinezza prebellica. [...] Tutti i nuovi scrittori poi hanno smarrito quella miracolosa immediatezza espressiva, quel nativo senso della terra e del reale, quella cruda saggezza che ci rese cari, a suo tempo, i Lee Masters, gli Hemingway, i Caldwell (Pavese 1962: 169).

Implícitamente, Pavese parece sugerir que el decenio de deslumbramiento por la cultura americana no ha sido archivado como una moda fugaz o una ingenuidad juvenil. Todo lo contrario: le queda la convicción del valor de, por lo menos, una docena de escritores - descendientes de los pioneros Whitman y Twain- que en un momento determinado contribuyeron lingüística y temáticamente - una nueva manera de contar- a renovar una tradición literaria cansada y asfixiada, como fue la italiana durante los años del fascismo:

Come era naturale in tempi di ristagno politico, noi tutti ci limitammo allora a studiare come quegli intellettuali d'oltremare avessero espresso questo dramma, 
come fossero giunti a parlare questo linguaggio, a narrare, a cantare questa favola (Pavese 1962: 175).

Este magisterio, es decir, la capacidad de poner en escena el "drama de todos", se ha desplazado, sencillamente, a otro lugar:

Noi abbiamo compreso che molti paesi dell'Europa e del mondo sono oggi il laboratorio dove si creano le forme e gli stili, e non c'è nulla che impedisca a chi abbia buona volontà, vivesse magari in un convento, di dire una nuova parola. Ma senza un fascismo a cui opporsi, senza un pensiero cioè storicamente progressivo da incarnare, anche l'America, per quanti grattacieli e automobili e soldati produca, non sarà più all'avanguardia di nessuna cultura. Senza un pensiero e senza una lotta progressiva, rischierà anzi di darsi essa stessa a un fascismo, e sia pure nel nome delle sue tradizioni migliori (Pavese 1962: 175).

Se encuentra en estas palabras, escritas en 1947, la alcanzada madurez de un escritor que ha meditado largamente sobre la obra ajena, para llegar a individuar un estilo y algunos temas propios y que se siente en condición, desde el "convento turinés", de decir, él también, una palabra nueva. El momento de pronunciarla llega después de unos años de búsquedas y después de que la experiencia americana le enseñara «che avere una tradizione è meno che nulla, è soltanto cercandola che si può viverla» (Melville 1995: 12). No nos parece un casualidad que aquella magistral obra de síntesis narrativa de un universo de sugestiones, experiencias, modos y reflexiones, que representa su última novela La luna e i falò, se abra, en inglés, con la frase Ripeness is all ${ }^{6}$, la madurez es todo.

Si algo no pudo prever Pavese fue, sin duda, la vigencia que el mito americano ha mantenido en Italia en la cultura popular, especialmente en la canción de unos cantautores que marcaron los años sesenta y setenta. El papel jugado por Fernanda Pivano fue, en este sentido, fundamental y empieza justamente a través de la amistad con Pavese, como ella misma cuenta:

Ero una ragazzina quando vidi per la prima volta l'Antologia di Spoon River: me l'aveva portata Cesare Pavese, una mattina che gli avevo chiesto che differenza c'è tra la lettura americana e quella inglese (Pivano 1971: xi).

Los primeros libros norteamericanos que Pavese facilitó a la joven Pivano, fueron tratados por ella «con grande sospetto» hasta el encuentro con la Antologia di Spoon River de Edgar Lee Master:

[...] l'aprii proprio alla metà, e trovai una poesia che finiva così «mentre la baciavo con l'anima sulle labra, l'anima d'improvviso mi fuggì». Chissà perché questi versi mi mozzarono il fiato: è così difficile spiegare le reazioni degli adolescenti (Pivano 1971: xii).

\footnotetext{
${ }^{6}$ Se trata de una cita de King Lear de Shakspeare.
} 
A lo largo del tiempo, la Antología de Spoon River se ha convertido para la cultura popular italiana en algo sorprendente, gracias a la publicación de un disco del cantautor Fabrizio De André, sin duda el más importante de su generación. En 1971 se publica, en efecto, Non al denaro, non all'amore, nè al cielo ${ }^{7}$, un disco libremente inspirado en la antología de Lee Master. De André eligió nueve de las 244 poesías y las transformó en canciones ${ }^{8}$, alterando en parte el texto, bajo la supervisión de la misma Fernanda Pivano.

Las nueve poesías seleccionadas tocan fundamentalmente dos grandes temas: la envidia (Un matto, Un giudice, Un blasfemo, Un malato di cuore) y la ciencia (Un medico, Un chimico, Un ottico $)^{9}$. Mientras en las poesías originales de Edgar Lee Masters cada personaje tiene un nombre y un apellido, los títulos de las canciones de De André son genéricos (un juez, un médico, etc.) para subrayar que las historias de estos personajes son ejemplos de comportamientos humanos que se pueden hallar en cada época y en cada lugar. La última canción, Il suonatore Jones ${ }^{10}$, es la única a la que De André deja el nombre original asignado por Lee Master.

En las notas que aparecen en el libreto del álbum se encuentra una interesante entrevista de Fernanda Pivano a De André; la misma Pivano escribió también para la ocasión una pseudoentrevista a Edgar Lee Masters. Aquí se aclara cómo todo el proyecto parece existir bajo la evocación de Cesare Pavese, empezando por el tema de la canción que abre el disco, la colina. Se trata, en efecto, de uno de los temas fundamentales en la obra del escritor piamontés y es el incipit tanto del libro, The Hill-La Collina, como del disco ${ }^{11}$.

Con respecto al trabajo de adaptación de los poemas de Lee Master, Pivano se expresó de manera entusiasta:

Fabrizio ha fatto un lavoro straordinario; lui ha praticamente riscritto queste poesie rendendole attuali, perché quelle di Masters erano legate ai problemi del suo tempo, cioè a molti decenni fa. Lui le ha fatte diventare attuali e naturalmente ha cambiato profondamente quello che era il testo originale; ma io sono contenta dei suoi cambiamenti e mi pare che lui abbia molto migliorato le poesie.

Sono molto più belle quelle di Fabrizio, ci tengo a sottolinearlo. Sia Masters che Fabrizio sono due grandi poeti, tutti e due pacifisti, tutti e due anarchici libertari,

\footnotetext{
7 DE ANDRÉ (1971).

8 Para una comparación entre los poemas de Masters, la traducción de Pivano, y los cambios aportados por De André, se remite a la página web de L. Masetti cit. en bibliografía.

9 En estos dos grupos se pueden descubrir simetrías: el juez, perseguido por todos, transforma su envidia en sed de poder y se venga; el químico está tan entregado a la ciencia y a la búsqueda de un orden perfecto que es incapaz de querer. El enfermo de corazón representa la alternativa a la envidia, que logra vencer gracias al amor en lugar de dejarse llevar por el egoísmo.

10 Sin duda, el músico Jones fue el personaje al que De André quiso compararse. Para Jones la música no es una profesión, es una elección de libertad; también De André, sobre todo en los últimos años de su carrera, ha tratado de desatarse de las limitaciones de la música como profesión, publicando sus últimos trabajos a una distancia de seis años uno del otro y reduciendo en público las apariciones.

11 Habla de toda aquella gente que como el músico Jones, «offrì la faccia al vento/la gola al vino e mai un pensiero/non al denaro, non all'amore né al cielo». Todos duermen ahora en la colina del cementerio de Spoon River.
} 
tutti e due evocatori di quelli che sono stati i nostri sogni. Poi Fabrizio sarà sempre attuale, è un poeta di una tale levatura che scavalca i secoli (Viva 2001: 152).

Curiosamente, la canción de autor y la poesía italiana nunca celebraron su encuentro, a diferencia de lo que ocurrió en otros países. Sobre este desencuentro no nos vamos aquí a pronunciar, simplemente constatamos que los cantautores italianos miraron hacia Estados Unidos y, de alguna manera, las razones que les animaron no fueron tan distantes de las de Pavese o Vittorini. Fue un furor, que llegaba con las protestas estudiantiles, con la beat generation y con los ejemplos de otros cantautores norteamericanos, fundamentalmente Bob Dylan. De esta relación musical y literaria cabe destacar, como hemos tenido modo de observar con el disco de De André, el papel jugado por Fernanda Pivano y, desde lejos, por Cesare Pavese.

\section{Bibliografía}

Amoruso, Vito (1960): «Cecchi, Vittorini e Pavese e la letteratura americana», Studi americani, n. 6, pp. 24-46.

D’Agostino, Nemi (1959): «Pavese e l'America», Studi americani, n. 4, pp. 89-102.

DE AnDRÉ, Fabrizio (1971): Non al denaro, non all'amore, nè al cielo. Milano, Ed. Musicali Ricordi.

DE ANDRÉ, Fabrizio; FASOLI, Doriano; CERI, Luciano (1996): Fabrizio De André: la cattiva strada: da Carlo Martello a don Raffaéy. Roma, Edizioni Associate Editrice Internazionale.

EsPOSITO, Edoardo (2004): Le letterature straniere nell'Italia tra le due guerre. Lecce, Pensa multimedia.

LAJOLO, Davide (1972): Il vizio assurdo: storia di Cesare Pavese. Milano, Mondadori.

LEE MASTERS, Edgar. (1971): Antologia di Spoon River. Torino, Einaudi.

LORENZI DAVITTI, Patrizia (1975): Pavese e la cultura americana: fra mito e razionalità. Messina, D'Anna.

MASETTI, Lorenzo (2002): L'antologia di Spoon River di Edgar Lee Master e Non al denaro, non all'amore, né al cielo di Fabrizio de André, en http://www.prato.linux.it/ lmasetti/percorsi_incrociati/spoonriver/index.php.

Melville, Herman (1995): Moby Dick. Torino, Einaudi.

PAVESE, Cesare (1952): Paesi tuoi. Torino, Einaudi.

PAVESE, Cesare (1969): La letteratura americana e altri saggi. Torino, Einaudi.

PAVESE, Cesare (1975): La luna e i falò. Torino, Einaudi.

PIVANO, Fernanda (1971): «Prefazione», en E. Lee Master, Antologia di Spoon River. Torino, Einaudi, pp. vi-xix.

PIVANO, Fernanda (2006): I miei amici cantautori. Milano, Mondadori.

TAPPARO, Elena (2006): Fernanda Pivano e la letteratura americana. Roma, Prospettiva.

VIVA, Luigi (2001): Non per un dio ma nemmeno per gioco: vita di Fabrizio De André. Milano, Feltrinelli.

VITTORINI, Elio (1968): Americana: Raccolta di narratori. Milano, Bombiani.

VITTORINI, Elio (1986): Conversazione in Sicilia. Milano, Rizzoli. 